\title{
Investment choices of individual investors in D.I.Khan, KPK province of Pakistan
}

\author{
Bela Kundi \\ (M.phil student, department of business administration/Gomal University D.I.Khan, Pakistan)
}

\begin{abstract}
The purpose of this study is to inquire the investment avenues of the people of D.I.Khan prefer to choose. Study showed that most of investors in the district prefer to deposit their savings at banks, post office savings \& real estate. Investment choices are many \& selection between them is based on investors' objectives. Income is key factor affect risk tolerance of investor.

Keywords - Investment Avenue, individual investor behaviour.
\end{abstract}

\section{INTRODUCTION}

Behavioural finance is an emerging science that studies the irrational behaviour of the people. Investment avenues are the investment choice which is available to the investor to invest depending upon their objective. Shares are more risky area but one can earn high return that will push up return of entire portfolio. A mutual fund is also an up-and-coming part for investment provides variety of scheme in market to suit the need of large group of investor in mutual fund like equity oriented finance. Risk is high but probability of high return is also high. Bonds are fixed-income securities because interest payment (coupon rate) \& principal repayment for a typical bond are specified at the time the bond is issued \& fixed for life of bond. Bond is (safe asset) in investors point of view. Principal \& interest is specified. Debenture is a type of debt instrument that is not covered by security of physical asset or collateral. Deposit at banks term as term deposits or fixed deposit. Minimum investment period is 30 days \& return rate varies with period of investment \&consider most liquidity item. Postal saving schemes low risk saving instrument. Insurance policy also promote saving \& additionally provide insurance coverage. Real-estate is investing in property, Agri-land, urban land; resorts etc. gold perform differently than other investment, provide all time return when on many occasion stock market fails

\section{LITERATURE REVIEW}

Chambers and Schlagenhauf, (2002); Gomes et al, (2004); Dynan et al, (2004) found that many factors can affect investments choice[1][2].Kapra, G., Mishra, P.K \& Dash M.K(2010) studied the investment behaviour \&perception of investment decision on the basis of age \& gender , he concluded age \& gender are the main variable which affect the risk taking capacity of investors[3]. Guiso, Japeelli\& Terlizzese(1996), Bajtelsmit,V.L., \& Bernasek, A.(1996), Jianakoplos \& Bernasek, A.(1999)Hariharan, Chapman \& $\operatorname{Domain}(\mathbf{2 0 0 0})$ found that woman are more risk averse than male respondent[4][5][6].

R. Kasilingam, Dr. G. Jayabal, uses demographic factors \& shows it impact on the portfolio choice under choice criteria, behavioural trait, source of information \& awareness level. He found that family size is unimportant but family income is key one, have an influence on the behaviour of investor[7]. N. Geetha, Dr M. Ramesh (2011) fined the relationship of Investment Avenue with gender \& age group[8]. Wiley \& chow (1992) find out investment decision is affected by income level \& return requirements. He found positive relationship between financial risk tolerance \& income[9]. Wallach, M.A \& Kogen, N (1961) found that risk taking capacity of investor is negatively related to marital status \& positively to age \& income[10]. Willem E. Frankenhuis, Johan C. Karremans (2012) do two experimental studies to find the risk taking preferences of single \& committed men. And found that involved male take less risk in the presence of women to the extent that they are more strongly committed to their current partners[11].

\section{RESEARCH METHODOLOGY}

The purpose of this paper is to predict the factors affecting investment choices of people of the city of D.I.Khan of KPK province of Pakistan. Study is based on primary data, by using questionnaire \& secondary data, by collecting data and information from various journals and reference books. First of all, investment choices are classified into 8 categories according to the answers to the question of

"Which investment option do you prefer to invest the major part of your savings?" as follows:

- $\quad$ cash at hand

- $\quad$ cash at bank

- $\quad$ insurance policy

- $\quad$ gold \& silver 
- $\quad$ Real estate(land)

- $\quad$ postal saving scheme

- $\quad$ bonds \&debenture

- $\quad$ shares \& mutual funds

The researcher categorized the respondents on basis of age, gender, income, occupation etc.

In case of Traders and Manufacturers, it includes all respondents engaged in small and medium trade like Jewellers, Computer Sales and Services, Booksellers, Building contractors, Hard ware merchants, manufacturer of coir products, steel tables and chairs, plastic products ,metal utensils, leather and rubber footwares, etc. Professionals includes Doctors, lawyers, Chartered Accountants, Tax consultants, Civil Engineers, Software and Mechanical Engineers etc.

The point of study is to conclude:

\section{OBJECTIVE OF STUDY:}

- Investment opportunities that people of D.I.Khan prefer.

- $\quad$ Factor influence investment behaviour of D.I.Khan district people.

- $\quad$ Source of information about financial instruments.

- Relationship between demographic factors of D.I.Khan district \& investment

avenues.

- Relationship risk acceptance to the income of D.I.Khan district people.

\section{Data Analysis}

In this analysis, people of D.I.Khan are giving preferences to cash at banks (23.5\%), gold \& silver (16\%), postal savings $(16 \%)$, insurance $(14.8 \%)$ \& real estate $(14.8 \%)$ which show their moderately conservative nature, to protect their principal, and achieve some real increase in the price of their investment. Second most preference to shares \&mutual funds (9.9\%) \& bonds \& debentures (3.7\%).

\section{V-1 Investment Avenue Regarding Gender Of Respondent:}

The distribution of respondent according to their gender \& is analyzed as presented in TableV-1

Tablev-1 INVESTMENT AVENUE REGARDING GENDER OF RESPONDENT:

\begin{tabular}{|c|c|c|c|c|c|c|c|c|c|}
\hline \multirow[b]{2}{*}{ gender } & \multicolumn{8}{|c|}{ Investment avenue } & \multirow[b]{2}{*}{ Total } \\
\hline & $\begin{array}{c}\text { cash } \\
\text { at } \\
\text { hand }\end{array}$ & $\begin{array}{c}\text { cash at } \\
\text { bank }\end{array}$ & $\begin{array}{l}\text { insurance } \\
\text { policy }\end{array}$ & $\begin{array}{l}\text { gold \& } \\
\text { silver }\end{array}$ & $\begin{array}{c}\text { Real } \\
\text { estate(land) }\end{array}$ & $\begin{array}{c}\text { postal } \\
\text { saving } \\
\text { scheme }\end{array}$ & $\begin{array}{c}\text { bonds } \\
\text { \&debenture }\end{array}$ & $\begin{array}{l}\text { shares } \\
\& \\
\text { mutual } \\
\text { funds }\end{array}$ & \\
\hline male & & $12.30 \%$ & $9.90 \%$ & $7.40 \%$ & $13.60 \%$ & $11.10 \%$ & $3.70 \%$ & $8.60 \%$ & $66.70 \%$ \\
\hline female & $1.20 \%$ & $11.10 \%$ & $4.90 \%$ & $8.60 \%$ & $1.20 \%$ & $4.90 \%$ & & $1.20 \%$ & $33.30 \%$ \\
\hline Total & $1.20 \%$ & $23.50 \%$ & $14.80 \%$ & $16.00 \%$ & $14.80 \%$ & $16.00 \%$ & $3.70 \%$ & $9.90 \%$ & $100.00 \%$ \\
\hline
\end{tabular}

Classification of respondent according to their gender is that $66.70 \%$ is male \& remaining is female. Male respondent prefer to invest in real estates, insurance, shares $\&$ mutual funds, bonds $\&$ debentures $\&$ also in bank deposit, which show their high risk taking behaviour. Where as large proportion of female prefers to invest in gold \& save money at banks which shows their risk adverse behaviour. Same result is graphically shown in figureV-1

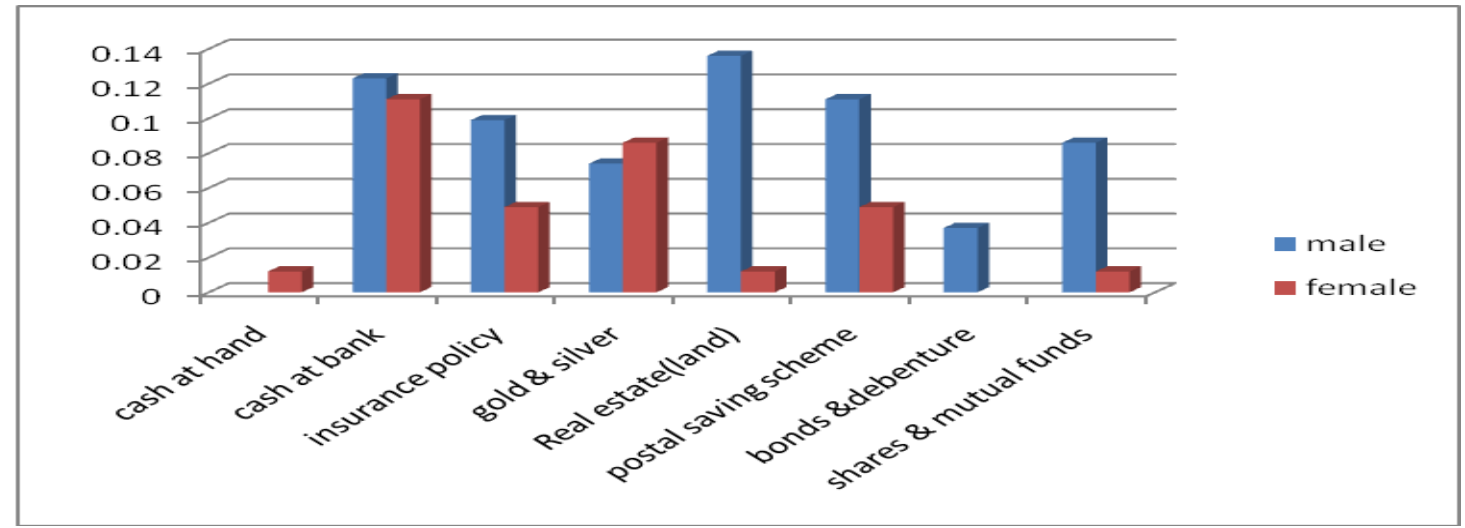

FigureV-1 INVESTMENT AVENUE REGARDING GENDER OF RESPONDENT: 


\section{Investment Avenue Regarding Age Of Respondent:}

The distribution of respondent according to their age \& is analyzed as presented in TableV-2

TableV-2 INVESTMENT AVENUE REGARDING AGE OF RESPONDENT:

\begin{tabular}{|l|c|c|c|c|c|c|c|c|c|}
\hline & \multicolumn{7}{|c|}{ investment avenue } & & \\
\hline age & $\begin{array}{c}\text { cash } \\
\text { at } \\
\text { hand }\end{array}$ & $\begin{array}{c}\text { cash at } \\
\text { bank }\end{array}$ & $\begin{array}{c}\text { insurance } \\
\text { policy }\end{array}$ & $\begin{array}{c}\text { gold \& } \\
\text { silver }\end{array}$ & $\begin{array}{c}\text { Real } \\
\text { estate(land) }\end{array}$ & $\begin{array}{c}\text { costal } \\
\text { saving } \\
\text { scheme }\end{array}$ & $\begin{array}{c}\text { shares } \\
\& \\
\text { bonds } \\
\text { \&utual } \\
\text { funds }\end{array}$ & Total \\
\hline $\begin{array}{l}\text { less } \\
\text { than } \\
25\end{array}$ & $1.20 \%$ & $3.70 \%$ & & $1.20 \%$ & & $1.20 \%$ & & $1.20 \%$ & $8.60 \%$ \\
\hline $\begin{array}{l}25 \\
35\end{array}$ & $11.10 \%$ & $7.40 \%$ & $6.20 \%$ & $9.90 \%$ & $2.50 \%$ & & $6.20 \%$ & $43.20 \%$ \\
\hline $\begin{array}{l}35 \text { to } \\
45\end{array}$ & & $4.90 \%$ & $7.40 \%$ & $6.20 \%$ & $4.90 \%$ & & $3.70 \%$ & $2.50 \%$ & $29.60 \%$ \\
\hline $\begin{array}{l}\text { above } \\
\text { than } \\
45\end{array}$ & & $3.70 \%$ & & $2.50 \%$ & & $12.30 \%$ & & & $18.50 \%$ \\
\hline Total & $1.20 \%$ & $23.50 \%$ & $14.80 \%$ & $16.00 \%$ & $14.80 \%$ & $16.00 \%$ & $3.70 \%$ & $9.90 \%$ & $100.00 \%$ \\
\hline
\end{tabular}

Age group of up to 35, gives preference in investing in cash at bank(11.10\%), Gold \& silver $(6.20 \%) \&$ real estates(9.90\%).\& age group of 36-45 gives preference to cash at bank(4.90\%),insurance policy $(7.40 \%)$,shares \& mutual funds $(2.5 \%)$. where as above 46 age group select cash at bank(3.70\%),\&postal saving(12.30\%)most preferably. Most of this sample prefers risk free or less risky avenues for investment also graphically shown in figureV-2

\section{Figure V-2 INVESTMENT AVENUE REGARDING AGE OF RESPONDENT}

Investment Avenue Regarding Marital-Status Of Respondent:

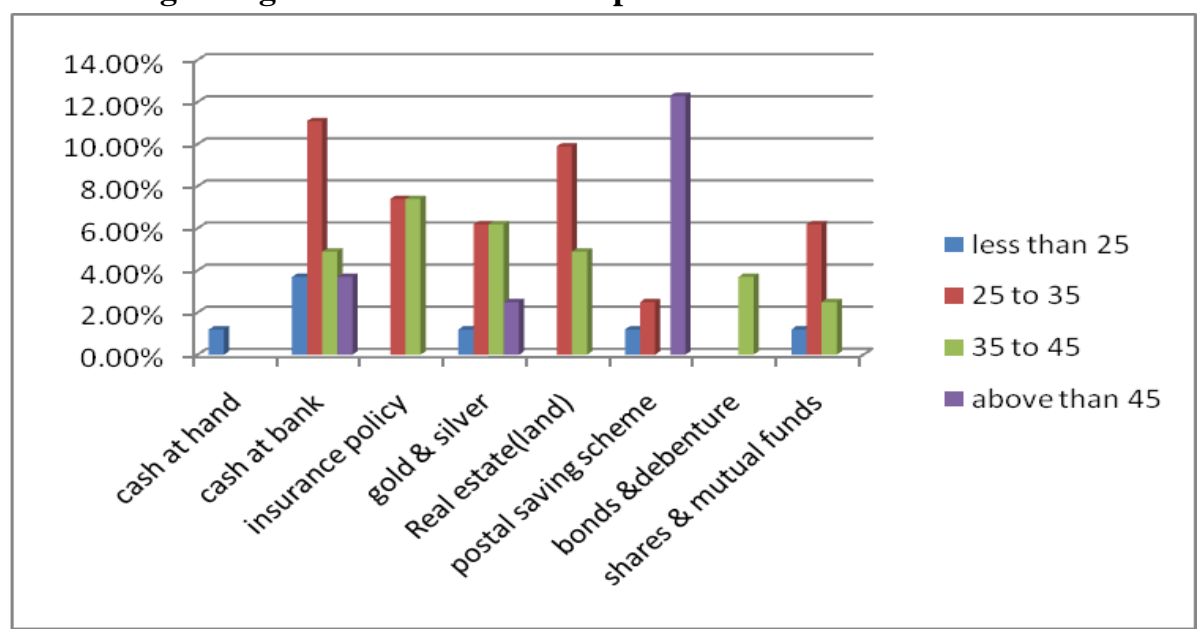

The distribution of respondent according to their marital status \& is analyzed as presented in Table V-3

Table V-3 INVESTMENT AVENUE REGARDING MARITAL-STATUS OF RESPONDENT:

\begin{tabular}{|c|c|c|c|c|c|c|c|c|c|}
\hline \multirow[b]{2}{*}{$\begin{array}{l}\text { marital } \\
\text { status }\end{array}$} & \multicolumn{8}{|c|}{ investment avenue } & \multirow[b]{2}{*}{ Total } \\
\hline & $\begin{array}{l}\text { cash } \\
\text { at } \\
\text { hand }\end{array}$ & $\begin{array}{c}\text { cash at } \\
\text { bank }\end{array}$ & $\begin{array}{c}\text { insurance } \\
\text { policy }\end{array}$ & $\begin{array}{l}\text { gold \& } \\
\text { silver }\end{array}$ & $\begin{array}{c}\text { Real } \\
\text { estate(land) }\end{array}$ & $\begin{array}{l}\text { postal } \\
\text { saving } \\
\text { scheme }\end{array}$ & $\begin{array}{c}\text { bonds } \\
\text { \&debenture }\end{array}$ & $\begin{array}{l}\text { shares } \\
\& \\
\text { mutual } \\
\text { funds }\end{array}$ & \\
\hline single & $1.20 \%$ & $11.10 \%$ & $7.40 \%$ & $6.20 \%$ & $4.90 \%$ & $1.20 \%$ & & $7.40 \%$ & $39.50 \%$ \\
\hline married & & $12.30 \%$ & $7.40 \%$ & $9.90 \%$ & $9.90 \%$ & $14.80 \%$ & $3.70 \%$ & $2.50 \%$ & $60.50 \%$ \\
\hline Total & $1.20 \%$ & $23.50 \%$ & $14.80 \%$ & $16.00 \%$ & $14.80 \%$ & $16.00 \%$ & $3.70 \%$ & $9.90 \%$ & $100.00 \%$ \\
\hline
\end{tabular}

Most of the single respondents give preference to shares \& mutual funds, cash in bank, insurance policy which shows that they (single) take risk by investing in shares \& mutual funds. Where as marries respondents give preference to cash at bank, insurance policy, gold \&silver \& real-estate which represents that they(married) try to invest in less risky investment cause of fear of loss. Respondent distribution marital-wise is graphically shown in figure $\mathrm{V}-3$ 
Figure V-3 INVESTMENT AVENUE REGARDING MARITAL-STATUS OF RESPONDENT:

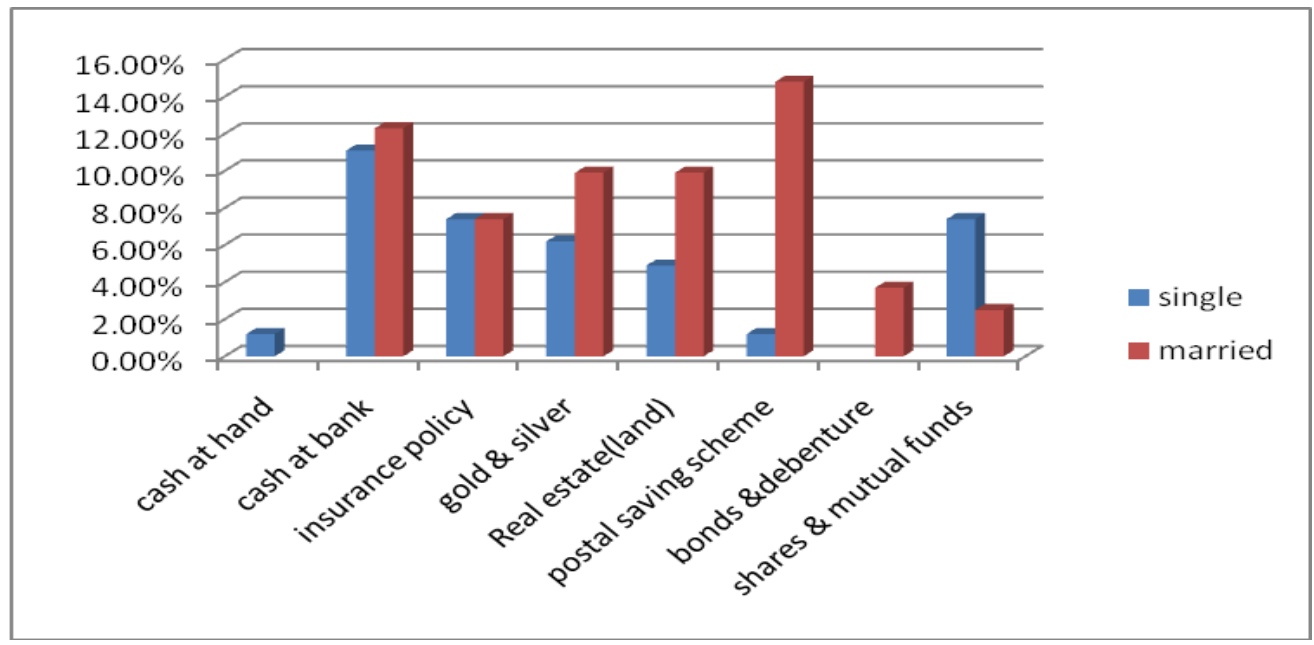

Investment Avenue Regarding Native Of Respondent:

The distribution of respondent according to their native $\&$ is analyzed as presented in TableV-4

Tablev-4 INVESTMENT AVENUE REGARDING NATIVE OF RESPONDENT:

\begin{tabular}{|c|c|c|c|c|c|c|c|c|c|}
\hline & \multicolumn{8}{|c|}{ investment avenue } & \\
\hline native & $\begin{array}{l}\text { cash } \\
\text { at } \\
\text { hand }\end{array}$ & $\begin{array}{l}\text { cash at } \\
\text { bank }\end{array}$ & $\begin{array}{l}\text { insurance } \\
\text { policy }\end{array}$ & $\begin{array}{l}\text { gold \& } \\
\text { silver }\end{array}$ & $\begin{array}{c}\text { Real } \\
\text { estate(land) }\end{array}$ & $\begin{array}{l}\text { postal } \\
\text { saving } \\
\text { scheme }\end{array}$ & $\begin{array}{c}\text { bonds } \\
\text { \&debenture }\end{array}$ & $\begin{array}{c}\text { shares } \\
\& \\
\text { mutual } \\
\text { funds }\end{array}$ & Total \\
\hline urban & $1.20 \%$ & $11.10 \%$ & $3.70 \%$ & $6.20 \%$ & $3.70 \%$ & $2.50 \%$ & $1.20 \%$ & $7.40 \%$ & $37 \%$ \\
\hline rural & & $12.30 \%$ & $11.10 \%$ & $9.90 \%$ & $11.10 \%$ & $13.60 \%$ & $2.50 \%$ & $2.5 \%$ & $63 \%$ \\
\hline Total & $1.20 \%$ & $23.50 \%$ & $14.80 \%$ & $16.00 \%$ & $14.80 \%$ & $16.00 \%$ & $3.70 \%$ & $9.90 \%$ & $100.00 \%$ \\
\hline
\end{tabular}

Distribution of respondent according of their native is $63 \%$ of investors belong to rural area of D.I.Khan district; they prefer to invest in real estate cash at banks, postal savings \& real estate. Where as remaining portion belong to urban area of this district, their preference avenue is shares \& mutual funds, insurance policy, gold \& silvers, bond \&debentures. Graphically distribution of respondent native-wise is shown in figureV-4

\section{NATIVE-WISE DISTRIBUTION OF REPONDENT}

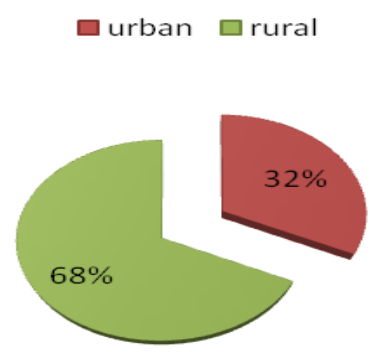

Figure V-4 INVESTMENT AVENUE REGARDING NATIVE OF RESPONDENT: 


\section{Investment Avenue Regarding Educational Qualification Of Respondent:}

The distribution of respondent according to their educational qualification \& is analyzed as presented in Table $\mathrm{V}-5$

Table V-5 INVESTMENT AVENUE REGARDING EDUCATIONAL QUALIFICATION OF RESPONDENT:

\begin{tabular}{|c|c|c|c|c|c|c|c|c|c|}
\hline \multirow[b]{2}{*}{$\begin{array}{l}\text { education } \\
\text { qualification }\end{array}$} & \multicolumn{8}{|c|}{ investment avenue } & \multirow[b]{2}{*}{ Total } \\
\hline & $\begin{array}{c}\text { cash } \\
\text { at } \\
\text { hand }\end{array}$ & $\begin{array}{c}\text { cash at } \\
\text { bank }\end{array}$ & $\begin{array}{l}\text { insurance } \\
\text { policy }\end{array}$ & $\begin{array}{l}\text { gold \& } \\
\text { silver }\end{array}$ & $\begin{array}{c}\text { Real } \\
\text { estate(land) }\end{array}$ & $\begin{array}{l}\text { postal } \\
\text { saving } \\
\text { scheme }\end{array}$ & $\begin{array}{c}\text { bonds } \\
\text { \&debenture }\end{array}$ & $\begin{array}{c}\text { shares } \\
\& \\
\text { mutual } \\
\text { funds }\end{array}$ & \\
\hline undergraduate & & $1.20 \%$ & $2.50 \%$ & $2.50 \%$ & $2.50 \%$ & $6.20 \%$ & & & $14.80 \%$ \\
\hline graduate & $1.20 \%$ & $18.50 \%$ & $11.10 \%$ & $12.30 \%$ & $8.60 \%$ & $9.90 \%$ & & $3.70 \%$ & $65.40 \%$ \\
\hline postgraduate & & $3.70 \%$ & $1.20 \%$ & $1.20 \%$ & $3.70 \%$ & & $3.70 \%$ & $3.70 \%$ & $17.30 \%$ \\
\hline doctorial & & & & & & & & $2.50 \%$ & $2.50 \%$ \\
\hline Total & $1.20 \%$ & $23.50 \%$ & $14.80 \%$ & $16.00 \%$ & $14.80 \%$ & $16.00 \%$ & $3.70 \%$ & $9.90 \%$ & $100.00 \%$ \\
\hline
\end{tabular}

Those of under graduate's respondent give first preference to postal savings \& second preference to Gold \& silver \& insurance \& real-estate. Graduates respondent like to block their savings by depositing money at banks\& secondly choice of to insurance policy. Postgraduates \& doctorate respondent of sample give prefer to invest money in shares\& mutual funds \& real estates. Result is graphically shown in figureV-5

\section{Figure V-5 INVESTMENT AVENUE REGARDING EDUCATIONAL QUALIFICATION OF RESPONDENT:}

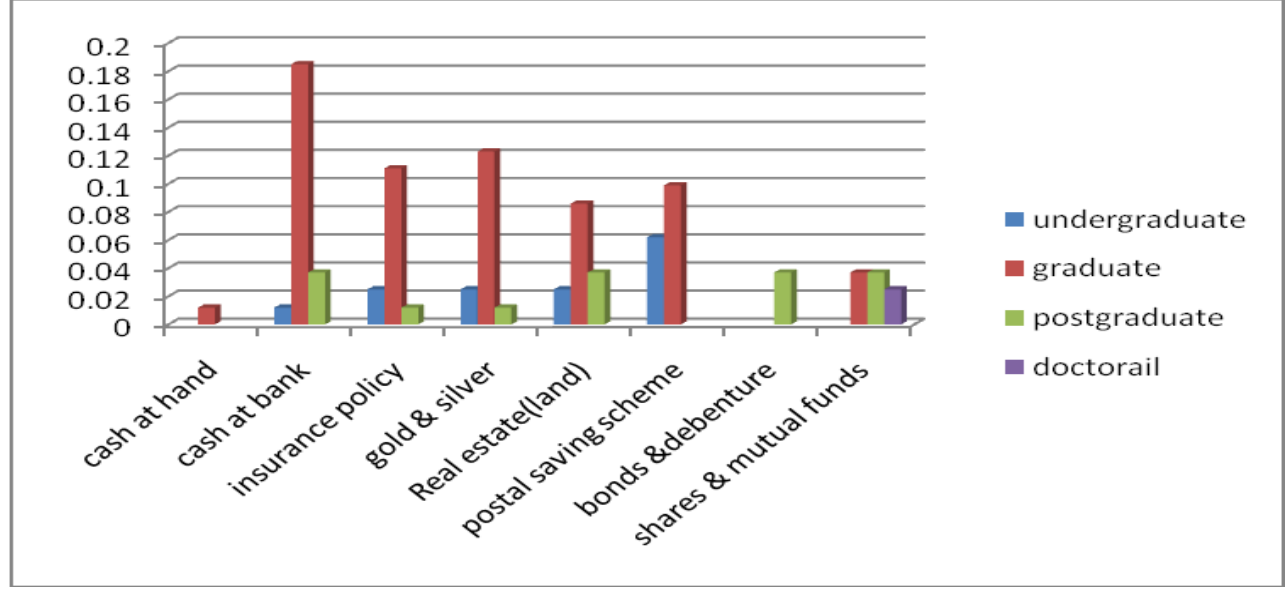

\section{Investment Avenue Regarding Occupation Of Respondent:}

The distribution of respondent according to their occupation \& is analyzed as presented in Table V-6

Table V-6 INVESTMENT AVENUE REGARDING OCCUPATION OF RESPONDENT:

\begin{tabular}{|c|c|c|c|c|c|c|c|c|c|}
\hline & \multicolumn{8}{|c|}{ investment avenue } & \multirow[b]{2}{*}{ Total } \\
\hline occupation & $\begin{array}{c}\text { cash } \\
\text { at } \\
\text { hand }\end{array}$ & $\begin{array}{l}\text { cash at } \\
\text { bank }\end{array}$ & $\begin{array}{l}\text { insurance } \\
\text { policy }\end{array}$ & $\begin{array}{l}\text { gold \& } \\
\text { silver }\end{array}$ & $\begin{array}{c}\text { Real } \\
\text { estate(land) }\end{array}$ & $\begin{array}{c}\text { postal } \\
\text { saving } \\
\text { scheme }\end{array}$ & $\begin{array}{c}\text { bonds } \\
\text { \&debenture }\end{array}$ & $\begin{array}{c}\text { shares } \\
\& \\
\text { mutual } \\
\text { funds }\end{array}$ & \\
\hline $\begin{array}{l}\text { trader,bussinessman } \\
\& \text { manufacturer }\end{array}$ & & $6.20 \%$ & $1.20 \%$ & & $4.90 \%$ & & & & $12.30 \%$ \\
\hline professional & & & & $3.70 \%$ & $2.50 \%$ & & $2.50 \%$ & $6.20 \%$ & $14.80 \%$ \\
\hline $\begin{array}{l}\text { salary earner-govt } \\
\text { sector }\end{array}$ & $1.20 \%$ & $12.30 \%$ & $6.20 \%$ & $7.40 \%$ & & $4.90 \%$ & $1.20 \%$ & & $33.30 \%$ \\
\hline $\begin{array}{l}\text { salary earner- } \\
\text { private sector }\end{array}$ & & $2.50 \%$ & $4.90 \%$ & $4.90 \%$ & $7.40 \%$ & $8.60 \%$ & & $3.70 \%$ & $32.10 \%$ \\
\hline others & & $2.50 \%$ & $2.50 \%$ & & & $2.50 \%$ & & & $7.40 \%$ \\
\hline Total & $1.20 \%$ & $23.50 \%$ & $14.80 \%$ & $16.00 \%$ & $14.80 \%$ & $16.00 \%$ & $3.70 \%$ & $9.90 \%$ & $100.00 \%$ \\
\hline
\end{tabular}

Salary earner (both private \& public) put their surplus money in banks \& postal savings, which shows their conservative behaviour toward risk. Professional class give preference to invest in shares $\&$ mutual funds, bonds \& debentures, which show their high-risk taking behaviour. Remaining category of occupational respondent give preference to real estate investment Avenue. . Result is graphically shown in figure V-6 
Figure V-6 INVESTMENT AVENUE REGARDING OCCUPATION OF RESPONDENT:

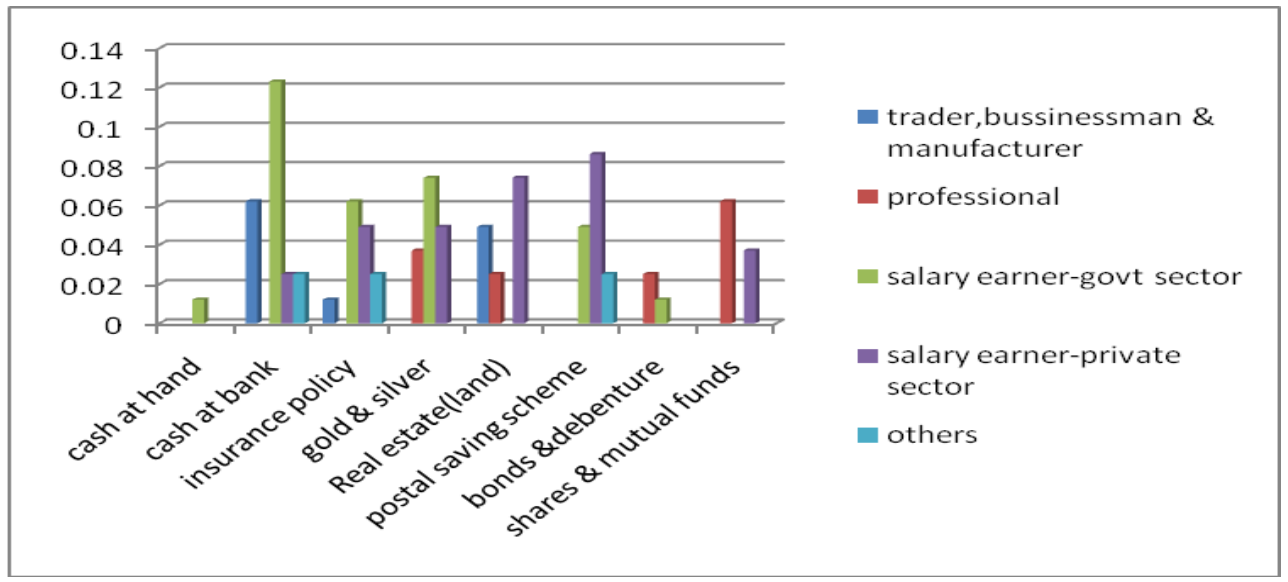

Investment Avenue Regarding Income (Monthly) Of Respondent:

The distribution of respondent according to their income (monthly) \& is analyzed as presented in TableV-7

TableV-7 INVESTMENT AVENUE REGARDING INCOME (MONTHLY) OF RESPONDENT:

\begin{tabular}{|c|c|c|c|c|c|c|c|c|c|}
\hline & \multicolumn{8}{|c|}{ investment avenue } & \multirow[b]{2}{*}{ Total } \\
\hline income & $\begin{array}{l}\text { cash } \\
\text { at } \\
\text { hand }\end{array}$ & $\begin{array}{c}\text { cash at } \\
\text { bank }\end{array}$ & $\begin{array}{c}\text { insurance } \\
\text { policy }\end{array}$ & $\begin{array}{l}\text { gold \& } \\
\text { silver }\end{array}$ & $\begin{array}{c}\text { Real } \\
\text { estate(land) }\end{array}$ & $\begin{array}{l}\text { postal } \\
\text { saving } \\
\text { scheme }\end{array}$ & $\begin{array}{c}\text { bonds } \\
\text { \&debenture }\end{array}$ & $\begin{array}{c}\text { shares } \\
\& \\
\text { mutual } \\
\text { funds }\end{array}$ & \\
\hline $\begin{array}{l}\text { below } \\
10000\end{array}$ & $1.20 \%$ & $3.70 \%$ & $1.20 \%$ & $4.90 \%$ & $1.20 \%$ & & & & $12.30 \%$ \\
\hline $\begin{array}{l}10000 \\
\text { to } \\
20000 \\
\end{array}$ & & $12.30 \%$ & $8.60 \%$ & $7.40 \%$ & $2.50 \%$ & $9.90 \%$ & & & $40.70 \%$ \\
\hline $\begin{array}{l}20000 \\
\text { to } \\
40000\end{array}$ & & $7.40 \%$ & $4.90 \%$ & $2.50 \%$ & $11.10 \%$ & $6.20 \%$ & $3.70 \%$ & $6.20 \%$ & $42.00 \%$ \\
\hline $\begin{array}{l}\text { above } \\
40000\end{array}$ & & & & $1.20 \%$ & & & & $3.70 \%$ & $4.90 \%$ \\
\hline Total & $1.20 \%$ & $23.50 \%$ & $14.80 \%$ & $16.00 \%$ & $14.80 \%$ & $16.00 \%$ & $3.70 \%$ & $9.90 \%$ & $100.00 \%$ \\
\hline
\end{tabular}

Lower income respondent give more to invest in cash at bank \& postal savings, insurance to save their money and get a fixed return with less chances of risk where as high income investors seek high risk and invest in shares \&mutual funds, bonds \& debenture \& real-estate. . Result of Investment Avenue is graphically shown in figureV-7

Figure V-7 INVESTMENT AVENUE REGARDING INCOME (MONTHLY) OF RESPONDENT:

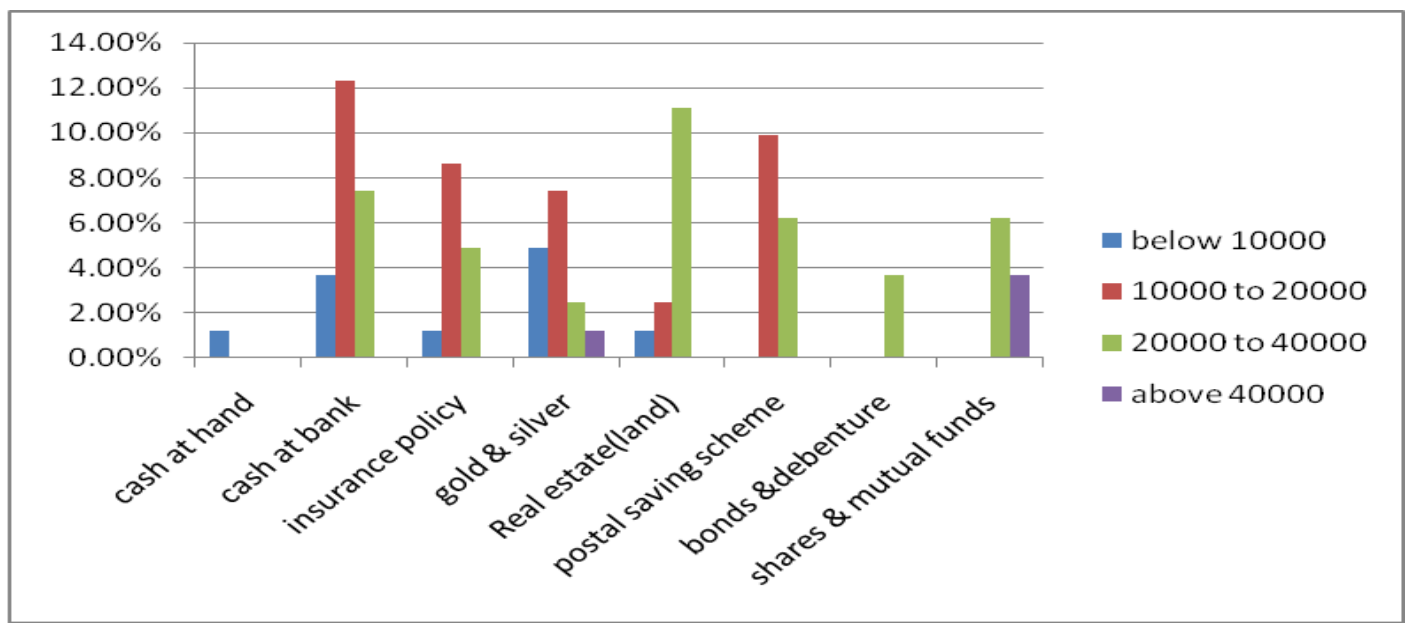




\section{Investment Avenue Regarding Saving (Monthly) Of Respondent:}

The distribution of respondent according to their saving (monthly) \& is analyzed as presented in Table V-8

Table V-8 INVESTMENT AVENUE REGARDING SAVING (MONTHLY) OF RESPONDENT

\begin{tabular}{|c|c|c|c|c|c|c|c|c|c|}
\hline \multirow[b]{2}{*}{ saving } & \multicolumn{8}{|c|}{ investment avenue } & \multirow[b]{2}{*}{ Total } \\
\hline & $\begin{array}{l}\text { cash } \\
\text { at } \\
\text { hand }\end{array}$ & $\begin{array}{l}\text { cash at } \\
\text { bank }\end{array}$ & $\begin{array}{l}\text { insurance } \\
\text { policy }\end{array}$ & $\begin{array}{l}\text { gold \& } \\
\text { silver }\end{array}$ & $\begin{array}{c}\text { Real } \\
\text { estate(land) }\end{array}$ & $\begin{array}{c}\text { postal } \\
\text { saving } \\
\text { scheme }\end{array}$ & $\begin{array}{c}\text { bonds } \\
\text { \&debenture }\end{array}$ & $\begin{array}{c}\text { shares } \\
\& \\
\text { mutual } \\
\text { funds }\end{array}$ & \\
\hline $\begin{array}{l}\text { below } \\
2000\end{array}$ & $1.20 \%$ & $12.30 \%$ & $3.70 \%$ & $6.20 \%$ & & $4.90 \%$ & $2.50 \%$ & & $30.90 \%$ \\
\hline $\begin{array}{l}2000 \\
\text { to } \\
5000\end{array}$ & & $6.20 \%$ & $9.90 \%$ & $8.60 \%$ & $9.90 \%$ & $4.90 \%$ & $1.20 \%$ & $2.50 \%$ & $43.20 \%$ \\
\hline $\begin{array}{l}5000 \\
\text { to } \\
10000\end{array}$ & & $3.70 \%$ & $1.20 \%$ & $1.20 \%$ & $4.90 \%$ & $6.20 \%$ & & $4.90 \%$ & $22.20 \%$ \\
\hline $\begin{array}{l}10000 \\
\text { to } \\
\text { above }\end{array}$ & & $1.20 \%$ & & & & & & $2.50 \%$ & $3.70 \%$ \\
\hline Total & $1.20 \%$ & $23.50 \%$ & $14.80 \%$ & $16.00 \%$ & $14.80 \%$ & $16.00 \%$ & $3.70 \%$ & $9.90 \%$ & $100.00 \%$ \\
\hline
\end{tabular}

$43.20 \%$ of the respondent saves up to 2000 to 5000, even they try to invest their money in Investment Avenue such as bank deposits, postal savings \& gold \& silver. Result is graphically shown in figure V-8 Figure V-8 INVESTMENT AVENUE REGARDING SAVING (MONTHLY) OF RESPONDENT

\section{Investment Objective Of Respondent:}

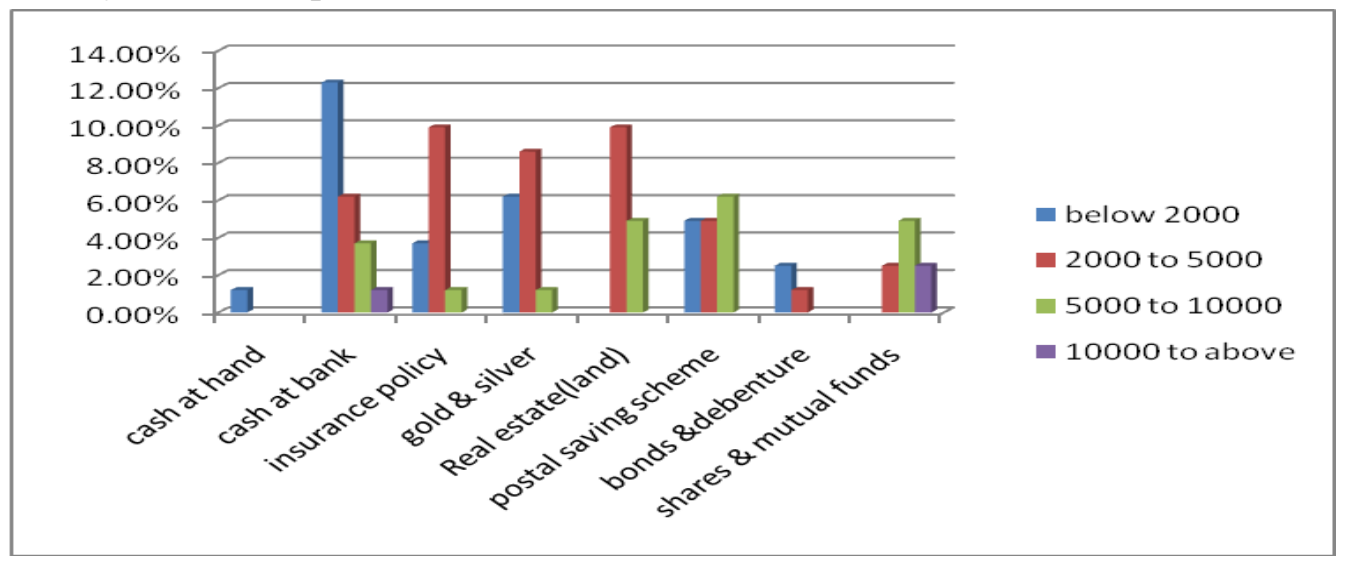

The distribution of respondent according to different investment objective $\&$ is analyzed as presented in Table V-9

Table V-9 INVESTMENT OBJECTIVE

\begin{tabular}{|l|r|r|}
\hline objective & $\begin{array}{l}\text { number of } \\
\text { respondent }\end{array}$ & \multicolumn{1}{l|}{ Total } \\
\hline high return & 30 & $37.00 \%$ \\
\hline safety/capital protection & 40 & $49.40 \%$ \\
\hline liquidity & 3 & $3.70 \%$ \\
\hline high growth & 8 & $9.90 \%$ \\
\hline Total & 81 & $100.00 \%$ \\
\hline
\end{tabular}




\section{OF RESPONDENT:}

Most of the respondent investment objective is to safety/capital protection. $37 \%$ of investor invest to earn high return as shown in figure V-9.

Figure V-9 INVESTMENT OBJECTIVE OF RESPONDENT:

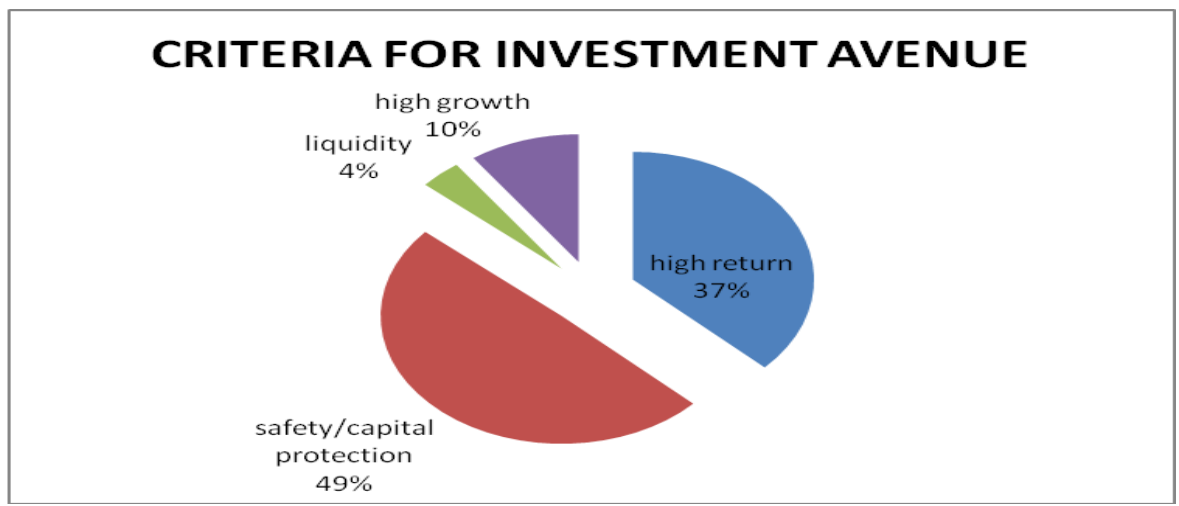

\section{INVESTMENT AWARNESS OF RESPONDENT:}

The distribution of respondent according to their awareness about different investment avenue $\&$ is analyzed as presented in Table V-10

Table V-10 INVESTMENT AWARNESS OF RESPONDENT:

\begin{tabular}{|r|r|r|}
\hline awareness & number of respondent & Total \\
\hline yes & 38 & $46.90 \%$ \\
\hline no & 43 & $53.10 \%$ \\
\hline Total & 81 & $100.00 \%$ \\
\hline
\end{tabular}

$53 \%$ of respondent have no awareness about different investment avenues, that's why they prefer bank deposit most then in postal savings \& real-estate.

\section{Source Of Investment Avenue Information:}

Investment information can be collect through different sources. On the basis of this information investor can made investment decision. There is several ways available to amass information, which are as shown in table V11 .

Table V-11 SOURCE OF INVESTMENT AVENUE INFORMATION:

\begin{tabular}{|r|r|r|}
\hline source of information & no. of respondent & percentage \\
\hline newspapers/ads/internet & 37 & $45.67 \%$ \\
\hline brokers/agent & 2 & $2.46 \%$ \\
\hline friend/relative & 26 & $32 \%$ \\
\hline financial consultant & 11 & $13.58 \%$ \\
\hline others & 5 & $6.17 \%$ \\
\hline total & 81 & $100 \%$ \\
\hline
\end{tabular}

$37 \%$ of respondent get information through newspapers/ads/internet. $26 \%$ of investor makes use of friend/relative confer information.

\section{Findings}

- Investors prefer where risk of loss is low \& have some return over it like by depositing in banks, or investing in real-estate etc, which show their moderately conservative nature, to protect their principal, and achieve some real increase in the price of their asset.

- Income of respondent is a key cause affects Investment Avenue \& their risk taking capability. Lower income group favour to invest in insurance bank deposit, post office savings rather than in other opportunity.

- Most of respondent prefer to invest for short time period (bank deposit).

- Sound financial background respondent invest in risky avenue.

- Young age respondent plan for wealth maximizations \& ready to put in in long term investments such as insurance policy, fixed deposit at bank.

- Aged respondent give preference to investment opportunity of regular frequent income. 
- Majority of investors have opted for bank deposit followed by insurance for investment purpose, then real estate .very few are interested in shares \&mutual funds, bond \& debentures.

- Respondents have medium knowledge about investment avenues and risk linked with it.

\section{Limitation Of Study}

Limited number of sample size \& reflect view of these respondents who are residing in D.I.Khan.I

\section{Reference}

[1]. Gomes, F., Alexander Michaelides, Valery Polkovnichenko; 2004. "Portfolio Choice and Wealth Accumulation With Taxable And Tax-Deferred Accounts”, September 2004, İnternet Adresi: Http:/Www.Cepr.Org/Pubs/Dps/Dp4852.Asp, (20.01.2010)

[2]. Chambers, M and D. E. Schlagenhauf; 2002. "Household Portfolio Allocations, Life Cycle Effects and Anticipated Inflation", Http://Garnet.Acns.Fsu.Edu/ Dschlage/Money.Pdf, (20.01.2010)

[3]. Kapra, G., Mishra, P.K \& Dash M.K(2010), "Factors influencing investment decision of generation in India:An Econometric study", Asain journal of management Research, pg 305-326

[4]. Guiso,L.,Jappelli,T., \& Terlizzese, D.(1996) income risk,borrowing constraints,and portfolio choice.american economic Review.86(1), 158-172

[5]. Bajtelsmit,V.L., \& Bernasek, A.(1996) Why do woman invest differently than Men? Financial Counseling \& Planning, 7,1-10.

[6]. Bajtelsmit,V.L., \& Bernasek, A. \& jianakoplos, N. A. (1999). Gender differences in defined contribution pebsion decisions.Financail Services Review, 8(1), 1-10.

[7]. R. Kasilingam, Dr. G. Jayabal, "impact of family size \&family income on the investment of salaried class investors, Ressek, pp93107.

[8]. N. Geetha, Dr M. Ramesh(2011) “A study on people's preferences in Investment Behaviour”IJEMR nov 2011 -Vol 1 issue 6.

[9]. Wallach, M.A \& Kogen, N.(1961) Aspect of judgement \& decision making irrelationships \& changes with age, Behavioural science, 6, 23-26.

[10]. Arms, Richard, trading without fear, john Wiley \& sons, 1992.

[11]. Willem E. Frankenhuis, Johan C. Karremans(2012) Uncommitted men match their risk taking to female preferences, while committed men do the opposite. Journal of experimental Social Psychology. Pp428-431.

[12]. Insights Into Investing in Gold, By Kevin Feldman, Managing Director of U.S. Marketing for iShares, Blackrock, T H E L O N D O N B U LL I O N M A R KET A S S C I A T I O N

[13]. Dynan, Karen, Jonathan Skinner and Stephen Zeldes; 2004. “Do The Rich Save More?” Journal of Political Economy, 112(2), (April), pp. 397-444 